\title{
Relationship between health status and religion in areas with high proportions of Christians in Sotome District, Nagasaki, Japan
}

\author{
Rieko NAKAO ${ }^{1}$, Kazuichi SUGIYAMA ${ }^{2}$, Akiko NITTA ${ }^{3}$ and Mayumi OHNISHI ${ }^{1}$
}

This study aimed to assess self-rated health (SRH) and self-rated mental health status (SRMH) among community dwellers in relation to individual religiosity and neighborhood religious characteristics and to consider factors associated with SRH and SRMH in Sotome District, Nagasaki, Japan. A comparison was made between the Kurosaki area, which has a high proportion of Christian residents, and the Konoura area, which has a proportion of Christian residents consistent with the Japanese average. An anonymous self-administered survey that collected data on sociodemographic information, religion, current medical history, self-rated economic satisfaction, SRH and SRMH was conducted in 2013. Comments about community activities, including religious activities, were obtained from leaders of residents' associations. A total of 424 (19.4\%) eligible residents living in the study area responded to the survey. After exclusion of responses with missing values, 287 (mean age 66.0 (standard deviation (SD) 14.0) years, range 30-91 years) responses were analyzed. The bivariate analysis showed that Christians from the Christian area were more likely than non-Christians to feel anxiety about the future (chi-square test, $P=0.001$ ). In the multivariate logistic regression analysis, the main factor associated with SRMH, which included anxiety (adjusted odds ratio (AOR): 3.74, 95\% confidence interval (CI): 2.01-6.94), helplessness (AOR: 3.97, 95\% CI: 2.20-7.17) and depression (AOR: 2.67, 95\% CI: 1.50-4.76), was poor self-rated economic status, regardless of individual religious faith or neighborhood religious characteristics. The main factor associated with mental health status in Sotome District was poor self-reported economic satisfaction. Interviews with the leaders of residents' associations revealed that Christian residents were more likely than non-Christian residents to be involved in religious and non-religious community activities. The mental health of residents in two regions with different neighborhood religious characteristics seems to be associated with self-rated economic satisfaction rather than religious factors.

Key words: Religion, Christians, self-rated health, self-rated mental health

\footnotetext{
${ }^{1}$ Department of Public Health Nursing, Nagasaki University Graduate School of Biomedical Sciences, Nagasaki, Japan

${ }^{2}$ Nagasaki University Graduate School of Fisheries Science and Environmental Studies, Nagasaki, Japan

${ }^{3}$ Nagasaki Wesleyan University, Nagasaki, Japan
} 


\section{Introduction}

Religiosity and/or spirituality are important factors related to health status ${ }^{1-3)}$, including physical health status, such as the presence of cardiovascular diseases ${ }^{4)}$, as well as mental status health ${ }^{5)}$ and quality of life $(\mathrm{QOL})^{6)}$. One study reported that the contributions of religiosity and spirituality are not the same because spirituality is associated with self-rated health and satisfaction, but religiosity is not $^{7)}$. However, how religiosity and/or spirituality contribute to health status and/or satisfaction has not been determined ${ }^{8,9)}$. In addition, gender differences in the relationships of religiosity with spirituality and health have been suggested, and the contributions of individual and public activities to health status and satisfaction may vary ${ }^{10,11)}$.

In general, Japan is considered a Buddhist and/ or Shintoist country. However, the study area in Sotome District near the western coast of Nagasaki Prefecture, Japan, has a particular historical and cultural background related to Christianity and/or KAKURE Christianity ("KAKURE" means "hidden" or "underground" in Japanese) because of the ban on the religion dating back to the $1600 \mathrm{~s}^{12,13)}$. In this study, the term "KAKURE Christians" refers not only to people who inherited Christianity in a unique form even after the Meiji era but also to people who practiced Christianity even during the ban $^{14)}$. Christianity was first introduced into Japan in the 16th century, and Nagasaki became the center of Japanese Christianity. However, during the Edo period (1603-1868), Christianity was persecuted, and some Christians continued to preserve Christianity by practicing their faith in secret without a priest as KAKURE Christians ${ }^{15)}$. Due to the influence of this historical background, in the areas of Kurosaki and Shitsu in Sotome District, the proportion of Christians is significantly higher than that in any other region in Japan, at approximately
60-70\%; the average proportion of Christians in the national Japanese population is approximately $1 \%$, and the average proportion in Nagasaki City is approximately $10 \%{ }^{16,17)}$. To this day, these areas are known to still be home to some KAKURE Christians. In contrast, in the area of Konoura in Sotome District, which is a neighboring area to Kurosaki and Shitsu, three temples exist. These temples were built during the ban period to spread Buddhism. Accordingly, most of the residents are Buddhists, and the proportion of Christians in the area of Konoura is similar to that in Nagasaki Prefecture, at approximately $4 \%{ }^{17)}$.

The health status of community dwellers was examined in two areas with different religious characteristics (Kurosaki and Konoura) in Sotome District. We describe the effect of religion on individual health status by comparing two neighboring areas with different religious characteristics. In addition, we consider religion at the individual level and examine whether neighborhood religious characteristics at the area level contribute to individual health status. This study aimed to assess self-rated mental health status $(\mathrm{SRMH})^{18)}$ and self-rated health $(\mathrm{SRH})^{19)}$ among residents in relation to individual religiosity and neighborhood religious characteristics and to consider factors associated with SRMH and SRH in Sotome District of Nagasaki, Japan.

\section{Methods}

\section{Study area}

This study was conducted in 2013 in Sotome District, Nagasaki Prefecture, Japan. The study was carried out by comparing two areas: the area of Kurosaki, as the Christian area, and the area of Konoura, as the non-Christian area.

\section{Study participants and data collection}

Among the residents' associations in Sotome district, ten residential wards (five from the Chris- 
tian area and five from the non-Christian area) that provided consent for this research were selected.

A self-administered anonymous questionnaire survey was conducted in these ten areas. The questionnaires were distributed to 664 households (472 households in the Christian area, 192 households in the non-Christian area) and collected from each household belonging to residents' associations through the leaders of the corresponding residents' associations. One member of each household completed the questionnaire after agreeing to participate in the survey.

In this study, we conducted interviews with all the leaders of the ten residents' associations to obtain comments about the study area in advance. The interviews included questions related to the religious affiliations of residents and participation in community activities, including religious activities.

\section{Measures}

The questionnaire included questions on sociodemographic information (age, sex, family structure, and occupation), current medical history, self-rated economic satisfaction, self-rated mental health status (SRMH), self-rated health (SRH), and individual religion. Individual's religion was selected from the choices of Christian, Buddhist or others. Individual religion was categorized into Christian and non-Christian according to the participants' responses regarding their religious faith. Non-Christian included Buddhists, those of other religious faiths, and those with no religious faith. Area religion, as an indicator of the neighborhood religious characteristics, was divided into Christian and non-Christian according to which of the major religious faiths was more prevalent in the communities. SRMH was measured using four questions from the mental health section of the QOL questionnaire developed by $\mathrm{Ohta}^{18)}$ for community-dwelling older adults: "Do you feel anxiety about the future?”, "Do you feel lonely?”, “Do you feel helpless?”, and "Do you feel depressed?”. Answers are given as "yes" or "no". Self-rated health was evaluated using a single question from the Comprehensive Survey of Living Conditions ${ }^{19)}$ : "How would you assess your health condition?" Answers were indicated on a four-point scale of "very good", "good", "fair", and "poor"; these responses were classified into two categories for statistical analysis: "very good" and "good" responses were classified as high self-rated health, and "fair" and "poor" responses were classified as low self-rated health.

\section{Data analysis}

The questionnaire data were analyzed by quantitative statistical methods. The chi-square test and Fisher's exact test were used to evaluate the associations between individual and area religion. After calculation of the crude odds ratio, logistic regression analysis was performed to determine factors associated with mental health and self-rated health. The statistical analyses were carried out using SPSS software version 24 for Windows. In all analyses, $\mathrm{p}<0.05$ was used to determine statistical significance. The interview data were summarized descriptively.

\section{Ethical considerations}

We explained the purpose of the study to the leaders of the residents' associations in each ward and obtained consent prior to performing data collection. Research consent was obtained in both paper and verbal form. A written explanation of the survey, including the study objectives and a guarantee of the voluntary nature of participation and privacy protection, was also distributed with the questionnaire. Submission of the completed questionnaire was recognized as consent to study participation from the residents. The study was approved by the ethics committees of the Nagasaki University Graduate School of Biomedical Sciences 
(approval number: 10081296).

\section{Results}

\section{Self-administered questionnaire survey with residents}

A total of 424 households (response rate (RR) $63.9 \%$ ), comprising 276 households in the Christian area (RR 58.5\%) and 148 households in the non-Christian area (RR 77.1\%) responded, accounting for approximately $80 \%$ of the 534 eligible households in the study area in 2013. One hundred thirty-seven respondents who did not provide their sex, age and/or individual religion were excluded from the analysis; thus, data from 287 study participants were analyzed (for a valid RR of $67.7 \%$ ). The mean respondent age was 66.0 (standard deviation; SD 14.0, rage; $30-91$ ) years. Table 1 shows the religious distribution of the study participants. Most of the respondents $(95,90.5 \%)$ from the non-Christian area were Buddhists, and only 3 (2.9\%) responded that they were Christians. On the other hand, 86 (47.3\%) respondents among the 182 respondents from the Christian area were Christians, and 75 (41.2\%) respondents were Buddhists (chi-'square test, $P<0.001)$.

Table 2 shows the sociodemographic characteristics of the study participants by individual religious faith and area religion; the data for three Christians in the non-Christian area were excluded. The participants from the non-Christian area were older than those from the Christian area (chisquare test, $P=0.008$ ), reflecting the demographic composition of the study area (proportion of population aged 65 years or over: $33.4 \%$ Christian area vs. $51.3 \%$ non-Christian area according to the Basic Resident Registration in 2010). In addition, the Christians from the Christian area reported the poorest self-rated economic satisfaction (chisquare test, $P<0.001$ ).

Table 3 shows the mental health and self-rated health by individual religion and area religion. Christians in the Christian area reported the highest anxiety of the three groups (chi-square test, $P=0.001)$. However, the univariate analysis showed no significant difference in loneliness, helplessness, depression, or self-rated health.

The factors associated with SRMH and SRH were analyzed by logistic regression analysis (Table 4). Poor self-rated economic satisfaction was associated with anxiety (adjusted odds ratio (AOR): 3.74, 95\% confidence interval (CI): 2.01-6.94), helplessness (AOR: 3.97, 95\% CI: 2.20-7.17), and depression (AOR: 2.67, 95\% CI: 1.50-4.76), regardless of individual religion and area religion. Female respondents were more likely to report depression than male respondents (AOR: $1.81,95 \%$ CI 1.023.22). Participants who lived alone (AOR: 2.28 , 95\% CI 1.09-4.76) and currently had diseases (AOR: 9.10, 95\% CI 3.59-23.08) were more likely to have low self-rated health.

Table 1 Religious distribution of the participants $(\mathrm{N}=287)$

\begin{tabular}{ccc}
\hline & $\begin{array}{c}\text { Christian Area } \\
\text { Kurosaki District }) \\
(\mathrm{n}=182)\end{array}$ & $\begin{array}{c}\text { Non-Christian Area } \\
\text { (Konoura District) } \\
(\mathrm{n}=105)\end{array}$ \\
\cline { 2 - 3 } & $\mathrm{n}(\%)$ & $\mathrm{n}(\%)$ \\
\hline Christians & $86(47.3 \%)$ & $3(2.9 \%)$ \\
Buddhists & $75(41.2 \%)$ & $95(90.5 \%)$ \\
Others & $21(11.5 \%)$ & $7(6.7 \%)$ \\
\hline
\end{tabular}

A chi- ${ }^{-}$square test was performed. 
Table 2 Sociodemographic characteristics of the study participants by individual religion and area religion $\left(\mathrm{N}=284^{*}\right)$

\begin{tabular}{|c|c|c|c|c|}
\hline & \multicolumn{2}{|c|}{ Christian area } & \multirow{2}{*}{$\begin{array}{c}\text { Non-Christian area } \\
\begin{array}{c}\text { Non-Christian } \\
(\mathrm{n}=102)\end{array} \\
\end{array}$} & \multirow{3}{*}{$P$-value } \\
\hline & $\begin{array}{c}\text { Christian } \\
(\mathrm{n}=86)\end{array}$ & $\begin{array}{c}\text { Non-Christian } \\
(\mathrm{n}=96)\end{array}$ & & \\
\hline & n (\%) & n (\%) & n (\%) & \\
\hline \multicolumn{5}{|l|}{ Sex } \\
\hline Male & $36(41.9)$ & $36(37.5)$ & $51(50.0)$ & \multirow{2}{*}{0.197} \\
\hline Female & $50(58.1)$ & $60(62.5)$ & $51(50.0)$ & \\
\hline \multicolumn{5}{|l|}{ Age (Median) } \\
\hline $30-67$ & $46(53.5)$ & $56(58.3)$ & 38 (37.3) & \multirow{2}{*}{0.008} \\
\hline $68^{-91}$ & $40(46.5)$ & $40(41.7)$ & $64(62.7)$ & \\
\hline \multicolumn{5}{|l|}{ Family structure } \\
\hline Living with someone & $54(79.4)$ & $51(61.4)$ & $67(70.5)$ & \multirow{2}{*}{0.056} \\
\hline Living alone & $14(20.6)$ & $32(38.6)$ & $28(29.5)$ & \\
\hline \multicolumn{5}{|l|}{ Job } \\
\hline Do not have a job/retired & 49 (60.5) & $47(54.0)$ & $61(63.5)$ & \multirow{2}{*}{0.413} \\
\hline Have a job & 32 (39.5) & $40(46.0)$ & 35 (36.5) & \\
\hline \multicolumn{5}{|l|}{ Self-rated economic satisfaction } \\
\hline Sufficient & $24(28.2)$ & $51(54.8)$ & $58(57.4)$ & \multirow{2}{*}{$<0.001$} \\
\hline Poor & $61(71.8)$ & $42(45.2)$ & $43(42.6)$ & \\
\hline \multicolumn{5}{|l|}{ Current diseases } \\
\hline No & $30(36.1)$ & $34(37.4)$ & $34(33.7)$ & \multirow{2}{*}{0.861} \\
\hline Yes & $53(63.9)$ & $57(62.6)$ & $67(66.3)$ & \\
\hline
\end{tabular}

A chi- ${ }^{-}$square test was performed.

*: Christians $(\mathrm{n}=3)$ in the non-Christian area were excluded from the analysis.

Table 3 Comparison of mental health and self-rated health by individual religion and area religion $\left(\mathrm{N}=284^{*}\right)$

\begin{tabular}{|c|c|c|c|c|}
\hline & \multicolumn{2}{|c|}{ Christian area } & \multirow{2}{*}{$\begin{array}{c}\text { Non }^{-} \text {Christian area } \\
\begin{array}{c}\text { Non-Christians } \\
(\mathrm{n}=102)\end{array}\end{array}$} & \multirow{3}{*}{$P$-value } \\
\hline & $\begin{array}{c}\text { Christians } \\
(\mathrm{n}=86)\end{array}$ & $\begin{array}{c}\text { Non-Christians } \\
(\mathrm{n}=96)\end{array}$ & & \\
\hline & n (\%) & n (\%) & n (\%) & \\
\hline \multicolumn{5}{|c|}{ Do you feel anxiety about the future? } \\
\hline Yes & $68(81.9)$ & $58(61.7)$ & $58(58.0)$ & \multirow{2}{*}{0.001} \\
\hline No & $15(18.1)$ & $36(38.3)$ & $42(42.0)$ & \\
\hline \multicolumn{5}{|l|}{ Do you feel lonely? } \\
\hline Yes & $34(40.5)$ & $41(45.1)$ & $45(44.6)$ & \multirow{2}{*}{0.799} \\
\hline No & $50(59.5)$ & $50(54.9)$ & $56(55.4)$ & \\
\hline \multicolumn{5}{|l|}{ Do you feel helpless? } \\
\hline Yes & $51(61.4)$ & $47(51.1)$ & $54(54.0)$ & \multirow{2}{*}{0.368} \\
\hline No & $32(38.6)$ & $45(48.9)$ & $46(46.0)$ & \\
\hline \multicolumn{5}{|c|}{ Do you feel depressed? } \\
\hline Yes & $46(55.4)$ & $52(57.1)$ & $53(53.5)$ & \multirow{2}{*}{0.882} \\
\hline No & $37(44.6)$ & $39(42.9)$ & $46(46.5)$ & \\
\hline \multicolumn{5}{|l|}{ Self-rated health } \\
\hline Very good/Good & $55(64.0)$ & $64(70.3)$ & $69(68.3)$ & \multirow{2}{*}{0.652} \\
\hline Fair/Poor & $31(36.0)$ & $27(29.7)$ & $32(31.7)$ & \\
\hline
\end{tabular}

A chi-square test was performed.

*: Christians $(n=3)$ in the non-Christian area were excluded from the analysis. 
Table 4 Factors associated with low mental health and self-rated health $\left(\mathrm{N}=284^{*}\right)$

\begin{tabular}{|c|c|c|c|c|c|c|c|c|c|c|}
\hline & \multicolumn{2}{|c|}{ Anxiety } & \multicolumn{2}{|c|}{ Loneliness } & \multicolumn{2}{|c|}{ Helplessness } & \multicolumn{2}{|c|}{ Depression } & \multicolumn{2}{|c|}{ Low self-rated health } \\
\hline & $\begin{array}{c}\text { OR } \\
(95 \% \mathrm{CI})\end{array}$ & $\begin{array}{c}\text { AOR } \\
(95 \% \mathrm{CI})\end{array}$ & $\begin{array}{c}\text { OR } \\
(95 \% \mathrm{CI})\end{array}$ & $\begin{array}{c}\text { AOR } \\
(95 \% \mathrm{CI})\end{array}$ & $\begin{array}{c}\text { OR } \\
(95 \% \mathrm{CI})\end{array}$ & $\begin{array}{c}\text { AOR } \\
(95 \% \mathrm{CI})\end{array}$ & $\begin{array}{c}\text { OR } \\
(95 \% \mathrm{CI})\end{array}$ & $\begin{array}{c}\text { AOR } \\
(95 \% \mathrm{CI})\end{array}$ & $\begin{array}{c}\text { OR } \\
(95 \% \mathrm{CI})\end{array}$ & $\begin{array}{c}\text { AOR } \\
(95 \% \mathrm{CI})\end{array}$ \\
\hline $\begin{array}{l}\text { Area religion } \\
\text { (ref. Non-Christian.) } \\
\text { Christian }\end{array}$ & $\begin{array}{c}1.79 \\
(1.07,2.99)\end{array}$ & $\begin{array}{c}0.79 \\
(0.40,1.58)\end{array}$ & $\begin{array}{c}0.93 \\
(0.57,1.53)\end{array}$ & $\begin{array}{c}1.11 \\
(0.58,2.13)\end{array}$ & $\begin{array}{c}1.08 \\
(0.66,1.78)\end{array}$ & $\begin{array}{c}0.87 \\
(0.44,1.71)\end{array}$ & $\begin{array}{c}1.12 \\
(0.68,1.83)\end{array}$ & $\begin{array}{c}1.05 \\
(0.54,2.04)\end{array}$ & $\begin{array}{c}1.05 \\
(0.62,1.77)\end{array}$ & $\begin{array}{c}1.02 \\
(0.47,2.23)\end{array}$ \\
\hline \multicolumn{11}{|l|}{$\begin{array}{l}\text { Individual religion } \\
\text { (ref. Non-Christian) }\end{array}$} \\
\hline Christian & $\begin{array}{c}3.05 \\
(1.63,5.72)\end{array}$ & $\begin{array}{c}2.17 \\
(0.94,4.99)\end{array}$ & $\begin{array}{c}0.84 \\
(0.50,1.41)\end{array}$ & $\begin{array}{c}0.69 \\
(0.33,1.42)\end{array}$ & $\begin{array}{c}1.44 \\
(0.85,2.43)\end{array}$ & $\begin{array}{c}1.29 \\
(0.61,2.76)\end{array}$ & $\begin{array}{c}1.01 \\
(0.60,1.70)\end{array}$ & $\begin{array}{c}0.72 \\
(0.34,1.50)\end{array}$ & $\begin{array}{c}1.27 \\
(0.74,2.17)\end{array}$ & $\begin{array}{c}1.27 \\
(0.54,2.96)\end{array}$ \\
\hline \multicolumn{11}{|l|}{ Sex (ref. Male) } \\
\hline Female & $\begin{array}{c}1.22 \\
(0.74,2.01)\end{array}$ & $\begin{array}{c}1.48 \\
(0.79,2.75)\end{array}$ & $\begin{array}{c}1.29 \\
(0.79,2.08)\end{array}$ & $\begin{array}{c}1.07 \\
(0.61,1.87)\end{array}$ & $\begin{array}{c}0.83 \\
(0.51,1.33)\end{array}$ & $\begin{array}{c}0.96 \\
(0.54,1.73)\end{array}$ & $\begin{array}{c}1.56 \\
(0.96,2.53)\end{array}$ & $\begin{array}{c}1.81 \\
(1.02,3.22)\end{array}$ & $\begin{array}{c}1.13 \\
(0.68,1.88)\end{array}$ & $\begin{array}{c}0.87 \\
(0.45,1.70)\end{array}$ \\
\hline \multicolumn{11}{|l|}{ Age (ref.30-67 y.) } \\
\hline $68^{-91 y}$ & $\begin{array}{c}0.68 \\
(0.41,1.12)\end{array}$ & $\begin{array}{c}0.50 \\
(0.23,1.07)\end{array}$ & $\begin{array}{c}1.26 \\
(0.78,2.02)\end{array}$ & $\begin{array}{c}1.25 \\
(0.64,2.43)\end{array}$ & $\begin{array}{c}1.63 \\
(1.01,2.63)\end{array}$ & $\begin{array}{c}1.49 \\
0.74,3.00\end{array}$ & $\begin{array}{c}0.83 \\
(0.52,1.34)\end{array}$ & $\begin{array}{c}0.70 \\
(0.35,1.40)\end{array}$ & $\begin{array}{c}2.14 \\
(1.28,3.58)\end{array}$ & $\begin{array}{c}1.20 \\
(0.56,2.56)\end{array}$ \\
\hline \multicolumn{11}{|c|}{$\begin{array}{l}\text { Family structure (ref. } \\
\text { Living with someone) }\end{array}$} \\
\hline Living alone & $\begin{array}{c}0.71 \\
(0.40,1.25)\end{array}$ & $\begin{array}{c}0.80 \\
(0.41,1.60)\end{array}$ & $\begin{array}{c}1.77 \\
(1.02,3.09)\end{array}$ & $\begin{array}{c}1.58 \\
(0.85,2.96)\end{array}$ & $\begin{array}{c}1.07 \\
(0.62,1.86)\end{array}$ & $\begin{array}{c}1.05 \\
(0.54,2.03)\end{array}$ & $\begin{array}{c}0.90 \\
(0.52,1.57)\end{array}$ & $\begin{array}{c}0.76 \\
(0.40,1.45)\end{array}$ & $\begin{array}{c}1.61 \\
(0.89,2.90)\end{array}$ & $\begin{array}{c}2.28 \\
(1.09,4.76)\end{array}$ \\
\hline \multicolumn{11}{|c|}{$\begin{array}{l}\text { Job (ref. Do not have a } \\
\text { job/retired) }\end{array}$} \\
\hline Have a job & $\begin{array}{c}1.17 \\
(0.69,1.98)\end{array}$ & $\begin{array}{c}0.71 \\
(0.34,1.50)\end{array}$ & $\begin{array}{c}0.68 \\
(0.41,1.13)\end{array}$ & $\begin{array}{c}0.77 \\
(0.39,1.51)\end{array}$ & $\begin{array}{c}0.74 \\
(0.45,1.21)\end{array}$ & $\begin{array}{c}0.82 \\
(0.41,1.64)\end{array}$ & $\begin{array}{c}0.87 \\
(0.53,1.44)\end{array}$ & $\begin{array}{c}0.73 \\
(0.36,1.45)\end{array}$ & $\begin{array}{c}0.58 \\
(0.33,1.00)\end{array}$ & $\begin{array}{c}1.29 \\
(0.57,2.92)\end{array}$ \\
\hline \multicolumn{11}{|c|}{$\begin{array}{l}\text { Self-rated economic sat- } \\
\text { isfaction(ref. Sufficient) }\end{array}$} \\
\hline Poor & $\begin{array}{c}3.82 \\
(2.25,6.50)\end{array}$ & $\begin{array}{c}3.74 \\
(2.01,6.94)\end{array}$ & $\begin{array}{c}1.46 \\
(0.90,2.36)\end{array}$ & $\begin{array}{c}1.63 \\
(0.93,2.86)\end{array}$ & $\begin{array}{c}3.05 \\
(1.86,5.01)\end{array}$ & $\begin{array}{c}3.97 \\
(2.20,7.17)\end{array}$ & $\begin{array}{c}2.12 \\
(1.31,3.46)\end{array}$ & $\begin{array}{c}2.67 \\
(1.50,4.76)\end{array}$ & $\begin{array}{c}1.55 \\
(0.93,2.60)\end{array}$ & $\begin{array}{c}1.61 \\
(0.84,3.10)\end{array}$ \\
\hline \multicolumn{11}{|l|}{$\begin{array}{l}\text { Current diseases } \\
\text { (ref. No) }\end{array}$} \\
\hline Yes & $\begin{array}{c}1.34 \\
(0.80,2.25)\end{array}$ & $\begin{array}{c}2.03 \\
(1.03,4.03)\end{array}$ & $\begin{array}{c}1.57 \\
(0.94,2.61)\end{array}$ & $\begin{array}{c}1.41 \\
(0.75,2.64)\end{array}$ & $\begin{array}{c}1.95 \\
(1.18,3.23)\end{array}$ & $\begin{array}{c}1.63 \\
(0.86,3.11)\end{array}$ & $\begin{array}{c}1.58 \\
(0.96,2.61)\end{array}$ & $\begin{array}{c}1.66 \\
(0.88,3.15)\end{array}$ & $\begin{array}{c}6.34 \\
(3.17,12.71)\end{array}$ & $\begin{array}{c}9.10 \\
(3.59,23.08)\end{array}$ \\
\hline
\end{tabular}

Logistic regression analysis was performed.

OR; Crude odds ratio, AOR; Adjusted odds ratio

*: Christians $(\mathrm{n}=3)$ in the non-Christian area were excluded from the analysis.

\section{Interviews with leaders of residents' associ- ation}

Table 5 shows community-based activities in the study areas, which were provided by the leaders of the residents' association. According to the estimations of the leaders of the residents' association, half of the residents identified themselves as Christian in the Christian area, while almost $100 \%$ of residents identified as Buddhist in the non-Christian area. In both the Christian area and non-Christian area, the residents were similarly involved in community activities such as sports festivals, beach cleanups, and traditional events. Additionally, the Christian area held a substantial number of church-related events (e.g., Sunday mass, cooking, meetings). Various meetings were held in churches, such as volunteer and women's club meetings. Statements given during the interviews in relation to religious events include the following:

"A Christian women's club is actively performed at the church regularly. The meetings usually include activities such as cooking, cleaning, and organizing bazaars."

"Even older people still come to church every Sun- 
Table 5 Summary of interviews with leaders of residents' associations in the study area

\begin{tabular}{|c|c|c|c|c|c|c|}
\hline \multirow{2}{*}{$\begin{array}{l}\text { Residents' } \\
\text { association }\end{array}$} & \multirow{2}{*}{$\begin{array}{l}\text { Households } \\
\text { number }^{\text {a }}\end{array}$} & \multirow{2}{*}{$\begin{array}{l}\text { Christian } \\
\text { residents }{ }^{b}\end{array}$} & \multirow{2}{*}{$\begin{array}{l}\text { Non-Cristian } \\
\text { residents }\end{array}$} & \multirow[b]{2}{*}{ Community activities } & \multicolumn{2}{|c|}{ Religious activities } \\
\hline & & & & & Related to Christianity & $\begin{array}{l}\text { Related to other } \\
\text { religion }\end{array}$ \\
\hline \multicolumn{7}{|c|}{ Christian area } \\
\hline $\mathrm{C}-1$ & 50 & $95 \%$ & $5 \%$ & $\begin{array}{l}\text { Sports festival, Mowing, Tradition- } \\
\text { al event (ONIBIYAKI and } \\
\text { whack-mole) }\end{array}$ & $\begin{array}{l}\text { Thanksgiving party, } \\
\text { Sunday mass }\end{array}$ & \\
\hline $\mathrm{C}-2$ & 64 & $50 \%$ & $50 \%$ & Sports festival, Cleanup activity & Christian meeting, & \\
\hline $\mathrm{C}-3$ & 60 & $20 \%$ & $80 \%$ & $\begin{array}{l}\text { New Year party, Traditional event } \\
\text { (ONIBIYAKI and whack-mole) }\end{array}$ & $\begin{array}{l}\text { Thanksgiving party, } \\
\text { Cultural festival }\end{array}$ & \\
\hline $\mathrm{C}-4$ & 75 & $50 \%$ & $50 \%$ & $\begin{array}{l}\text { Sports recreation, Dinner party, } \\
\text { Mowing, Beach cleanup }\end{array}$ & $\begin{array}{l}\text { Church meeting, } \\
\text { Women's club, } \\
\text { Volunteer activities, } \\
\text { Cleanup of church, } \\
\text { Cooking }\end{array}$ & \\
\hline $\mathrm{C}^{-} 5$ & 93 & $70 \%$ & $30 \%$ & $\begin{array}{l}\text { Bus tour for older people, Sports } \\
\text { festival, Mowing, Beach cleanup, } \\
\text { Volunteer activities }\end{array}$ & Sunday mass & Bon festival \\
\hline \multicolumn{7}{|c|}{ Non-Christian area } \\
\hline$N^{-1}$ & 42 & $0 \%$ & $100 \%$ & $\begin{array}{l}\text { Sports festival, Beach cleanup, Din- } \\
\text { ner party }\end{array}$ & & Shrine festival \\
\hline $\mathrm{N}-2$ & 45 & $8 \%$ & $92 \%$ & $\begin{array}{l}\text { Sports festival, New Year party, } \\
\text { KARAOKE, Ground golf, Mowing }\end{array}$ & & $\begin{array}{l}\text { Shrine festival, Bon } \\
\text { festival }\end{array}$ \\
\hline $\mathrm{N}-3$ & 31 & $0 \%$ & $100 \%$ & Sports festival, Beach cleanup & & Temple events \\
\hline $\mathrm{N}-4$ & 30 & $0 \%$ & $100 \%$ & $\begin{array}{l}\text { Sports festival, Ground golf, } \\
\text { Mowing, Traditional event (ONIBI- } \\
\text { YAKI) }\end{array}$ & & Shrine festival \\
\hline $\mathrm{N}-5$ & 44 & Not r & ported $^{\mathrm{c}}$ & $\begin{array}{l}\text { Sports festival, KARAOKE, Ground } \\
\text { golf, Cherry blossoms viewing } \\
\text { party, Cleanup activity, Mowing }\end{array}$ & & $\begin{array}{l}\text { Shrine festival, } \\
\text { Cleanup of shrine }\end{array}$ \\
\hline
\end{tabular}

a: Data from the Basic Resident Registration in 2010, b. Estimation according to the leader of the residents' association, c: The leader of the residents' association did not want to respond.

day morning mass. So, I can always say hello or ask them about their health condition."

"Church meetings are held at local community centers. Then, many of the activities of residents' associations are performed in a Christian church."

On the other hand, residents in the non-Christian area have only a few opportunities for religious activities. Statements given during interviews in relation to religious events include the following:

"Few local events for residents are held at temples." "A lot of people belonging to those temples as parishioners live outside of the district."

"Due to the aging community residents and the declining number of residents, community activities or events cannot be held."

\section{Discussion}

The bivariate analysis showed that Christians from the Christian area were more likely to feel anxiety about the future, and this finding received some support from the multivariate logistic regression analysis. Both individual Christians and the Christian area had higher crude ORs, but not adjusted ORs, of anxiety. However, there was a consistent association between poor self-rated economic satisfaction which seemed in many Christians and poor mental health status. This suggested that self-rated economic satisfaction was confounded. The main factor associated with low mental health status, which included anxiety, help- 
lessness, and depression, was poor self-rated economic status. The relationship between low income and poor health status has been reported in previous studies ${ }^{20-22)}$. In particular, the relationship between poverty or low income and depression was noted as a problem for vulnerable people $\mathrm{e}^{23,24)}$. The subjective assessment of low economic satisfaction of the Christians in the Christian area might be associated with poor mental health. In addition, the depression tendency of middle-aged women was shown to be associated with low economic status in a previous study ${ }^{25)}$, and the same tendency was observed in this study.

The factors associated with low SRH were living alone and currently being ill. Previous studies reported that SRH is an indicator that evaluates not only physical health but also social health status $^{26,27)}$. In this study as well, SRH was associated with both physical and social factors.

Although the dominant religions in Japan are Buddhism and/or Shintoism, a considerable number of people report having no religion ${ }^{28)}$. In general, studies conducted in Japan have not included religion as a socioeconomic factor ${ }^{29)}$, while international studies have considered the importance of religiosity and/or spirituality ${ }^{30)}$. Therefore, there are few studies on the relation between health and religion in Japan. In this study, respondents who indicated having an "other religion" probably actually practiced no religion because it is extremely rare for Japanese people to be Muslims or practice other religions. Furthermore, KAKURE Christians may have selected the "other religion" response because they did not belong to the Catholic Church and did not identify as Catholic. Conceivably, they were included among the non-respondents. One hundred thirty ${ }^{-}$seven non-respondents who were excluded from the analysis included 53 who did not respond to the individual religion question, and they could also be considered to be KAKURE
Christian or to have no religion. Therefore, the health of KAKURE Christians was not reported in a previous study and could not be fully clarified in this study.

Christians from the Christian area were more likely to report poor economic satisfaction and low mental health status, but non-Christians from the Christian area and non-Christians from the non-Christian area did not show the same tendency. Christians from the Christian area might have had particular characteristic feelings and ways of thinking regardless of their socioeconomic conditions. The authors could not fully determine the influence of the Christian participants' own characteristics on their mental health statuses based on the findings of this study. However, interviews with leaders of residents' associations suggested that Christians participate in more religious activities than non-Christians. Interactions through religious activities might be associated with the mental health of Christians from the Christian area. Previous research revealed that church-based social relationships are not always beneficial ${ }^{31,32)}$ and that close interaction with fellow church members may become a source of conflict, depression, or death ideation $^{33,34)}$. Alternatively, poor mental health might stimulate close interaction in religious activities. Therefore, participation in religious activities might be associated with poor mental health.

There are several limitations in this study. First, the findings from this study could not provide reasonable explanations for lower SRH and SRMH according to the neighborhood religion or individual religious faith, especially because few Christians resided in the non-Christian area. Although a continuous study to reveal the details of the factors associated with poor health conditions among Christians in the Christian area should conducted, the potential presence of particular conditions relating 
to health status among this population is important to consider. Second, there may be selection bias in the sample, because the study was conducted in only two areas, and the questionnaires were distributed to and collected from each household by the leaders of the corresponding residents' associations. Nevertheless, this study can provide valuable information regarding the characteristics related to health conditions among Christians from a Christian area in Japan.

\section{Conclusions}

Compared to non-Christians in both the Christian and non-Christian areas, Christians in the Christian area had lower mental health statuses in terms of their anxiety about the future. The main factor associated with mental health status was self-reported poor economic satisfaction. Interviews with the leaders of residents' associations revealed that Christian residents were more likely to be involved in religious and non-religious community activities than non-Christian residents. The mental health of residents in two regions with different neighborhood religious characteristics seems to be associated with self-rated economic satisfaction rather than religious factors.

\section{Acknowledgments:}

The authors are grateful to all of the community dwellers for their participation in this study and to the community leaders who collaborated in this study.

\section{Conflict of interest statement (for all authors):}

The authors have no competing interests to declare.

\section{Funding or sources of support in the form of grants, equipment, drugs, etc.:}

This study was supported by Nagasaki University. However, the content of this study is the sole responsibility of the authors and does not represent the official views of Nagasaki University.

\section{References}

1) Seeman TE, Dubin LF, Seeman M. Religiosity/spirituality and health. A critical review of the evidence for biological pathways. Am Psychol, 2003; 58(1): 53-63.

2) Mishra SK, Togneri E, Tripathi B, Trikamji, B. Spirituality and religiosity and its role in health and diseases. J Relig Health, 2017; 56(4): 1282-1301.

3) VanderWeele TJ. Religion and health in Europe: cultures, countries, context. Eur J Epidemiol, 2017; 32(10): 857-861.

4) Powell LH, Shahabi L, Thoresen CE. Religion and spirituality. Linkages to physical health. Am Psychol, 2003; 58(1): 36-52.

5) Koenig HG. Research on religion, spirituality, and mental health: a review. Can J Psychiatry, 2009; 54(5): 283-91.

6) Lim JW, Yi J. The effects of religiosity, spirituality, and social support on quality of life: a comparison between Korean American and Korean breast and gynecologic cancer survivors. Oncol Nurs Forrum, 2009; 36(6): 699-708.

7) Dankulincova VZ, Jirasek I, Veselsky P, et al. Spirituality but not religiosity is associated with better health and higher life satisfaction among adolescents. Int J Environ Res Public Health, 2017; 15(12). pii: E2781. doi: 10.3390/ijerph15122781.

8) Zimmer Z, Jagger C , Chiu C T , et al. Spirituality, religiosity, aging and health in global perspective: A review. SSM Popul Health, 2016; 2: 373-381.

9) Sugioka Y. Scientific researches about the impact of spirituality on health. J Intl Soc life Info Sci, 2012; 30(1): 94-105.

10) Maselko J, Kubzansky LD. Gender differences in religious practices, spiritual experiences and health: results from the US General Social Survey. Soc Sci Med, 2006; 62 (11): 2848-60.

11) Imamura $\mathrm{E}$, Loveland-Cherry $\mathrm{CJ}$, Whall AL, Relationships among age, gender, and religiosity in elder Japanese. Journal of Graduate School of Nurs- 
ing, Chiba University, 2016; 38: 11-20.

12) Harrington AM. The Kakure Kirishitan and their place in Japan's religious tradition. Japanese Journal of Religious Studies 7/4, 1980; 318-336.

13) Kakure Kirishitan. Downes P, Catholic Education Resource Center. https://www.catholiceducation. org/en/culture/catholic ${ }^{-}$contributions/kakure- $^{-}$ kirishitan.html (accessed November 27, 2020)

14) Miyazaki K. Kakure Kirishitan. Tokyo, Japan: KADOKAWA Corp, pp21, 2018. (In Japanese)

15) Miyazaki K. What did the latent Christian believe?. Tokyo, Japan: KADOKAWA Corp, 2018. (In Japanese)

16) Shukyo Nenkan (Religious Yearbook). Japanese Agency for Cultural Affairs. https://www.bunka. go.jp/tokei_hakusho_shuppan/hakusho_ nenjihokokusho/shukyo_nenkan/pdf/h26nenkan. pdf (accessed September 27, 2020)

17) Statistics of the Catholic Church in Japan 2016. Catholic Bishops' Conference of Japan. https:// www.cbcj.catholic.jp/japan/statistics/ (accessed November 27, 2020)

18) Ohta $T$, Haga H, Osada $H$, et al. Development and evaluation of a QOL questionnaire for elderly subject living in a community. J Jpn J Public Health, 2001; 48(4): 258-267 (In Japanese).

19) Ministry of Health, Labour and Welfare. Comprehensive Survey of Living Conditions 2018, (In Japanese). https://www.mhlw.go.jp/toukei/saikin/hw/ k-tyosa/k-tyosa19/dl/04.pdf (accessed November 27, 2020).

20) Social determinants of health: the solid facts, 2nd edition. WHO regional office Europe, edited Wilkinson, R., \& Marmot, M. 2003. http://www. euro.who.int/_data/assets/pdf_file/0005/98438/ e81384.pdf\#search=\%27the+solid+facts\%27 (accessed November 27, 2020).

21) Li-Fan L, Pei-Fang S. What factors influence healthy ageing? A person-centered approach among older adults in Taiwan. Geriatr Gerontol Int, 2017; 17: 697-707.

22) Sasaki I, Kondo K, Kondo N, et al. Are pension types associated with happiness in Japanese older people?: JAGES cross-sectional study. PLoS ONE, 2018; 13(5): e0197423. doi: 10.1371/journal.pone. 0197423.

23) Haseda M, Kondo N, Ashida T, et al. Community social capital, built environment, and income- based inequality in depressive symptoms among older people in Japan: An ecological study from the JAGES project. J Epidemiol, 2018; 28(3): 108-116.

24) Mendenhall M, Kohrt AK, Norris SA, et al. Non-communicable disease syndemics: poverty, depression, and diabetes among low-income populations. Lancet, 2017; 389(10072): 951-963. doi 10.1016/S0140-6736(17)30402-6.

25) Nakao R, Honda S, Moji K, Abe Y, Aoyagi, K. Relationship between lifestyle and mental health: A population-based study in Nagasaki prefecture, Japan. Acta Med Nagasaki, 2011; 55(2): 55-60.

26) Nishi A, Kawachi I, Shirai K, et al. Sex/gender and socioeconomic differences in predictive ability of self-rated health for mortality. Pros ONE, 2012; 7(1): e30179. doi: 10.1371/journal.pone. 0030179

27) Wei S, Watanabe M, Tanimoto Y, et al. Factors associated with good self-rated health of non-disabled elderly living alone in Japan: a cross-sectional study. BMC Public Health, 2007; 7: 297. doi: 10.1186/1471-2458-7-297

28) Nishi K, Survey of Japanese religions. The NHK Monthly Report on Broadcast Research, 2009;May: 66-81 (In Japanese). https://www.nhk.or.jp/ bunken/summary/research/report/2009_05/ 090505.pdf (accessed November 27, 2020).

29) Nagamine Y, Kondo N, Yokobayashi K, et al. Socioeconomic disparity in the prevalence of objectively evaluated diabetes among older Japanese adults: JAGES cross ${ }^{-}$sectional data in 2010. J Epidemiol, 2019; 29 (8): 295-301. doi: 10.2188/jea.JE20170206

30) Tsunoda H, Yoshino R, Yokoyama K. Components of social capital and socio-psychological factors that worsen the perceived health of Japanese males and females. Tohoku J Exp Med, 2008; 216(2): 173-85.

31) Neal K. Explorring the relationships among humility, negative interaction in the church and depressed affect. Aging \& Mental Health, 2014; 18(8): 970-979.

32) Danille RJ, Erin K P, Ryan DG, Kelly C. The moderating effect of the negative impact of recent life events on the relation between intrinsic religiosity and death ideation in older adults. Suicide and Life-Threatening Behavior, 2012; 42(6): 589-601. Doi: 10.1111/j.1943-278X.2012.00114.x

(Received 6.27.2020 ; Accepted 1.16.2021) 


\title{
Relationship between health status and religion in areas with high proportions of Christians in Sotome District, Nagasaki, Japan
}

\author{
Rieko NAKAO, Kazuichi SUGIYAMA, Akiko NITTA and Mayumi OHNISHI
}

Jpn J Health \& Human Ecology, 87 (6) 255-264, 2021

和文抄録

【目的】長崎県外海地区における住民の精神的健康および主観的健康感と地域の宗教的背景および 個人の宗教を含めた社会人口学的要因との関連について評価する.

【方法】住民にキリスト教徒の割合が高い黒崎地域（キリスト教地域）とキリスト教徒の割合が長 崎県の值と同レベルの神浦地域（非キリスト教地域）とを比較した。住民に自治会を通じて無記 名自記式質問紙調査を実施した。調査項目は，回答者の基本属性と宗教，現病歴，精神的健康， 主観的健康感であった。同時に，両地域において宗教的な活動を含む地域活動について自治会長 から意見を聞いた。

【結果】総数 424 人から質問紙調査の回答が得られ，性別や年齢，宗教が未回答であった 137 人を 削除した 287 人を分析対象とした。自身がキリスト教徒であると回答した割合はキリスト教地域 では 86 名 $(47.3 \%)$ であり，非キリスト教地域では 3 名 $(2.9 \%)$ であった。単変量分析では, キ リスト教地域のキリスト教徒が非キリスト教徒よりも将来への不安を感じていた（カイ二乗検定， $\mathrm{p}=0.001$. ロ ジスティック回帰分析の結果では，地域の宗教的背景や個人の宗教との関連は見られ ず，主観的な経済ゆとり満足感の低さが将来への不安（調整オッズ比（AOR）：3.74,95\%信頼区間 (Cl) : 2.01-6.94), 無力感 ( $\mathrm{AOR}: 3.97,95 \% \mathrm{CI}: 2.20-7.17$ ), うつ傾向 ( $\mathrm{AOR}: 2.67,95 \% \mathrm{CI}: 1.50^{-}$ 4.76）と関連していた。主観的健康感の低さには，独居であること（AOR：2.28, 95\% CI : $1.09-$ 4.76), 現病があること（AOR：9.10,95\%CI：3.59-23.08）が関連していた。自治会長のインタ ビューからキリスト教徒のほうが宗教的な活動を含む地域での活動が活発であることが推察され た.

【結論】長崎県外海地区の地域の宗教的背景が異なる 2 つの地域の住民の精神的健康には宗教的な 要因ではなく，主観的な経済ゆとり満足感の低さとの関連が示された。主観的健康感には，独居 と現病といった個人的な要因が関連していた。 